Proceedings of the 2002 IEEE Intemational

Conterence on Control Applications

September 18-20, 2002 • Glasgow, Scotland, U.K.

\title{
CONDITIONAL INTEGRATION ON PID FOR TYPES 1 AND 2 CONTROL SYSTEMS
}

\author{
Ramón Ferreiro García and Francisco Javier Pérez Castelo \\ Departamento Ingeniería Industrial. Universidad de La Coruña \\ E.S Marina Civil, Paseo de Ronda 51- 15011 La Coruña (Spain). e-mail: ferreiro@udc.es
}

\begin{abstract}
In this work some practical aspects of PI(D) controllers regarding to conditional integration are described. This contribution concerns to the integral action of a PID controller, which is managed as function of set-point changes and control error sign changes. The consequence derived from the contribution based in conditional integration (CI) of the PID integral action when set-point and error sign changes, is a drastic reduction in response overshoot to command inputs. Such contribution is successfully applied to control systems that belong to type 1 and type 2 systems, among which, are ship steering control and aircraft vehicles control.
\end{abstract}

Keywords: Integral action, conditional integration, clearing integral action, overshoot suppression,

\section{INTRODUCTION TO CONDITIONAL INTEGRATION AND ANTIRESET WINDUP.}

Some practical aspects of PI(D) controllers deal with non-linear constraints inherent to the basic control actions. The integral action of every practical PI(D) controller is restricted or limited to prevent reset windup. When a controller with integral action, PI or PID sees an error signal for a long period of time, it integrates the error until it reaches a maximum, usually $100 \%$ of scale or a minimum, $0 \%$ of scale (Astrom. Karl. J,Wittenmark B 1984). This is called reset windup.

In software based controllers the integration action is turned off when the controller does not have control of the valve or its associated final control element. In conclusion, a regulator with integral action is an input output unstable system. Its unstable mode can give rise to difficulties under certain circumstances.

Reset windup or integrator saturation can occur if the output saturates and the controller continues to integrate the error. The output of the integrator can then assume very large values, and it can take a long time to get it back to a normal value again. This problem is automatically avoided when the velocity form of the algorithm is used, because the integration stops automatically. When the output is limited. If it is desirable to use the position form, then some precaution must be taken. One way is to stop updating the integrator when the output is limited.

The strategy of turning off integration when the controller output is limited may be complemented to improve performance in special cases of track-keeping control by means of a conditional integration algorithm (R. Ferreiro García. A. Ameal. 2001).

To the well stabilised methods with its advantages of managing reset windup, a complement to this algorithm based in the use of conditional integration is proposed. In this complement to reset windup, the integral part of the controller is cancelled when control error sign changes due to an external command input. Such contribution is specially useful in track-keeping control.

\section{A COMPLEMENT TO RESET WINDUP: CI ALGORITHM}

In the case of reset windup controllers, the output of the integrator can then assume only normal values restricted to high and low limits. In process control that belong to type 1 or type 2 systems, it is common the case in which such a controller is operating into the high or low limits when the error approach or becomes zero or error sign is changing. In both cases, to get the controller action in the right direction due to the time to reduce the integral value from the actual limit to zero value, it take some time. During this time, the system response is not properly (oscillation), and the performance is poor. This problem is efficiently avoided by setting the integral value to zero under some conditions. That means the actual integration 
value will be set to zero when error sign is changing (only once per sign change). This task is a conditional integration and as it consists in clearing the integral value, it will be called Clear Integration (CI) algorithm

That basic idea consists in clearing the integral action value at any instant for which error sign changes. The effect of such strategy is that the time necessary to decrease the integration value is set to zero, and consequently, controller efficiency is better in some practical cases.

Several alternative CI algorithms may be implemented. The algorithms depend on the method used to perform the integral action but in all of them the following rule must be applied:

IF Error sign change under step setpoint changes THEN Reset integration value.

Consequently, the following steps must be performed:

- Setpoint changes detection

- Error sign changes detection

- Reset Integration Action

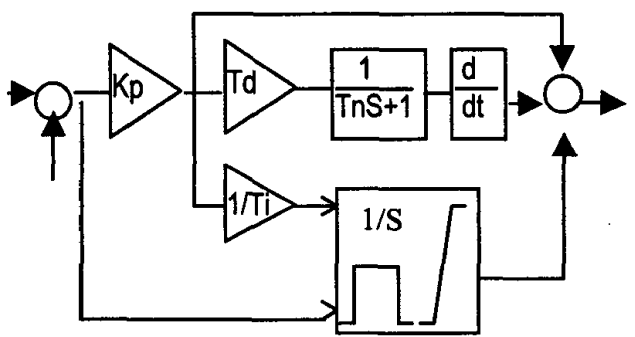

Fig. 1. Implementation to clear integration action in a PID controller

Implementation of proposed $\mathrm{CI}$ algorithm might be carried out by means of Simulink. In figure 4 it is shown a PI controller equipped with such CI algorithm. The integrator block is specially useful to perform conditional integration under error change of sign.

\section{TYPICAL APPLICATIONS}

Candidate applications to proposed controller are all type 1 and type 2 systems under track-keeping mode. Among the processes of type 1 or type 2 but not limited to the following are:
- Ship positioning

- Ship steering

- Aircraft steering, including satellites and missiles controls

- Aircraft roll and pitch control, including missiles.

- Aircraft's autopilot.

- Terrain vehicles positioning like underground or rail train among other special vehicles.

The typical structure of such kind of controlled system is shown in figure 2 in which the feedback control of a type 1 system is represented.

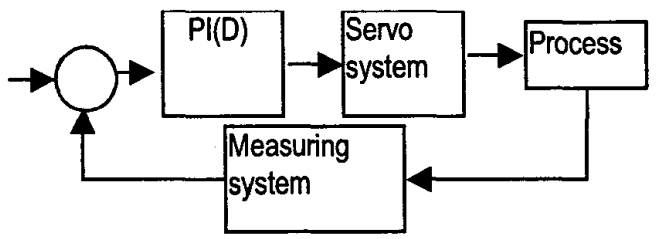

Fig. 2. Block diagram of the type 1 system under a feedback structure.

The strategy based in conditional integration by $\mathrm{CI}$ which consists in clearing integral action under changes of control error sign, becomes especially useful to reduce drastically or avoid excessive response overshoot to set point changes or command inputs. Such strategy might be used continuously or be alternatively activated when command changes are detected, being cancelled when steady state is reached.

\section{VALIDATION OF CI ALGORITHM}

This section is dedicated to validate the proposed contribution. Validation of $\mathrm{CI}$ algorithm will be carried out $\mathrm{n}$ the basis of simulation results. Two standard useful models are used. One of them belong to a beam rider missile and a second one belong to the roll motion control of an aircraft.

Both models have been researched on the basis of dynamic simulation tests to compare the responses between conventional and proposed PID.

\section{$A$ beam rider missile}

A semiautomatic antitank missile requires the gunner to track the target with a sight that projects a beam of 
light onto the tank (Ollis Rubin 1986) The missile has sensors that measure its position with reference to the axis of this beam, and its autopilot causes it to fly along the beam. The objective of the guidance system is to manoeuvre the missile to correct for any observed deviations from the beam. The missile is manoeuvred by servomotors, which deflect its elevator control surfaces. The aerodynamic forces on these surfaces produce moments that pitch the missile to an angle of attack, causing lift on its wings. This causes, in turn, the missile to manoeuvre by lateral acceleration. The overall weapon system is shown in figure 3 . The pitch control system for missile guidance is shown in figure 4 , where a linear model comprising a amplifier, a motor, the elevator and an inertial load that belong to a type 2 system is also included.

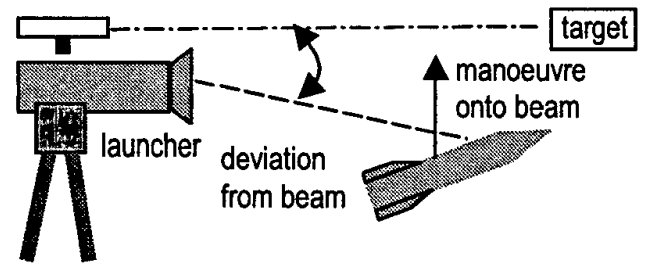

Fig. 3. The weapon system

Figure 4 shows the pitch motion model of a missile control system

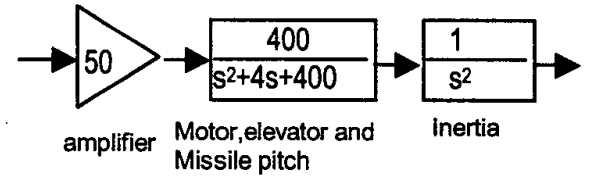

Fig. 4. The model for a missile pitch control system

\section{Roll control of an aircraft}

The aircraft model is used to simulate the roll control behaviour, comparing the response to command inputs in three conditions: with a conventional reset windup PID controller, with a reset windup plus $\mathrm{CI}$ controller and with a lead predictor compensator properly adjusted. In both cases, results are shown.

An aircraft will fly straight when its wings are level, and will turn if it is banked (Ollis Rubin 1986). Consider the design of an autopilot that will control this bank angle as measured by an artificial horizon. The aircraft is controlled by means of servomotors, which differentially deflects the aileron control surfaces. The aerodynamic forces on these surfaces will then produce rolling moments on the body. In this application, we shall only consider the design of the autopilot under one flight condition that means constant speed and altitude.

In the aircraft model, it is assumed that the aileron deflection produces a proportional sustained roll rate of 10 degrees/s per degree, while the inertia of aircraft causes its roll to lag behind this with a time constant of $0.5 \mathrm{~s}$. The servo amplifier voltage produces a servomotor torque, giving a proportional aileron deflection of 1 degree/ $\mathrm{V}$, and the torque lags behind the voltage by a time constant of $0.075 \mathrm{~s}$. The inertia of the aileron causes its dynamic response to be oscillatory, having comparable behaviour to that of a weathercock that swings about the wind. The natural frequency is $11 \mathrm{rad} / \mathrm{s}$ and damping ratio is 0.9 .

The navigation system flies the aircraft along a beam towards a radio beacon by turning the aircraft to reduce any deviations from the beam. The roll autopilot, which responds to bank angle commands from the navigation system, is shown in figure 5.

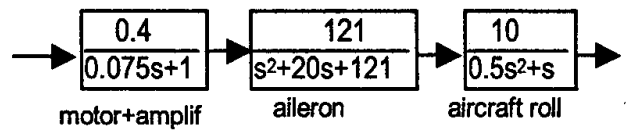

Fig. 5. The model of an aircraft roll control.

\subsection{The results by simulation}

In this subsection some graphical results are presented. Such results belong to both models described by figures 4 and 5 .

The weapon system shown in figure 3 is simulated using the model of a missile pitch control system shown in figure 4. A feedback control structure is used to compare responses with a conventional reset windup PID controller and a conventional reset windup plus CI algorithm PID controller. Comparison of both results are shown in figure 6. Under a set-point change applied to the feedback controlled system, with and without CI algorithm, responses are clearly different. The performance is better with the addition of proposed $\mathrm{CI}$ algorithm. 
The aircraft roll control system shown in figure 5 is simulated using the model of the aircraft roll motion. A feedback control structure is used to compare responses with a conventional reset windup PID controller, a conventional reset windup plus $\mathrm{CI}$ algorithm PID controller and a classic lead predictor compensator. Comparison of both results are shown in figure 7. Under a set-point change applied to the feedback controlled system, with and without $\mathrm{Cl}$ algorithm, responses are clearly different. The response due to the lead predictor is satisfactory but performance will not be sufficient because the lack of disturbances rejection capacity The performance is better with the addition of proposed CI algorithm because the integral action contributes to increase low frequency gain which is necessary to improve disturbance rejection.

\section{MAIN RESULTS AND CONCLUSIONS}

An exhaustive series of test have been carried out on proposed models. The total amount of tests related to such cases respond with a clear advantageous differences, regarding conventional reset windup: overshoot is drastically reduced or avoided in practical terms.

Simulation results presented by means of figures 6 and 7 , shows that for all studied cases, performance with CI algorithm is better than using only conventional reset windup PI(D) controllers. The most relevant conclusions regarding the analysis studied cases following to step heading changes, are:

- For all tests, steering performance using CI algorithm, is better reducing overshoot.

- The possibility of apply the PI(D) controller in different processes requires the implementation of a selector to apply $\mathrm{CI}$ algorithm depending on the system type. $(0,1,2)$.

\section{REFERENCES}

Astrom. Karl. J,Wittenmark B(1984).Computer controlled systems Ed. Prentice-Hall Inc, Englewood Cliffs, N.J.07631. USA

Chap. 8. pp. 181-186

Astrom. K.J. Wittenmark B. 1989. Adaptive Control. Ed. Addison-Wesley Publishing.
Chap 10, pp. 355-359.

Ollis Rubin (1986). The design of automatic control systems. Ed. Artech House. Inc, 685 Canton Street, Norwood, MA 02062. USA. Chap. 9, pp. 192-199

R. Ferreiro García. A. Ameal. (2001). CAMS'01 High performance $P I(D)$ controllers using an alternative to reset windup. Univ. Of Starthclytde, Gasgow, Scotland. 


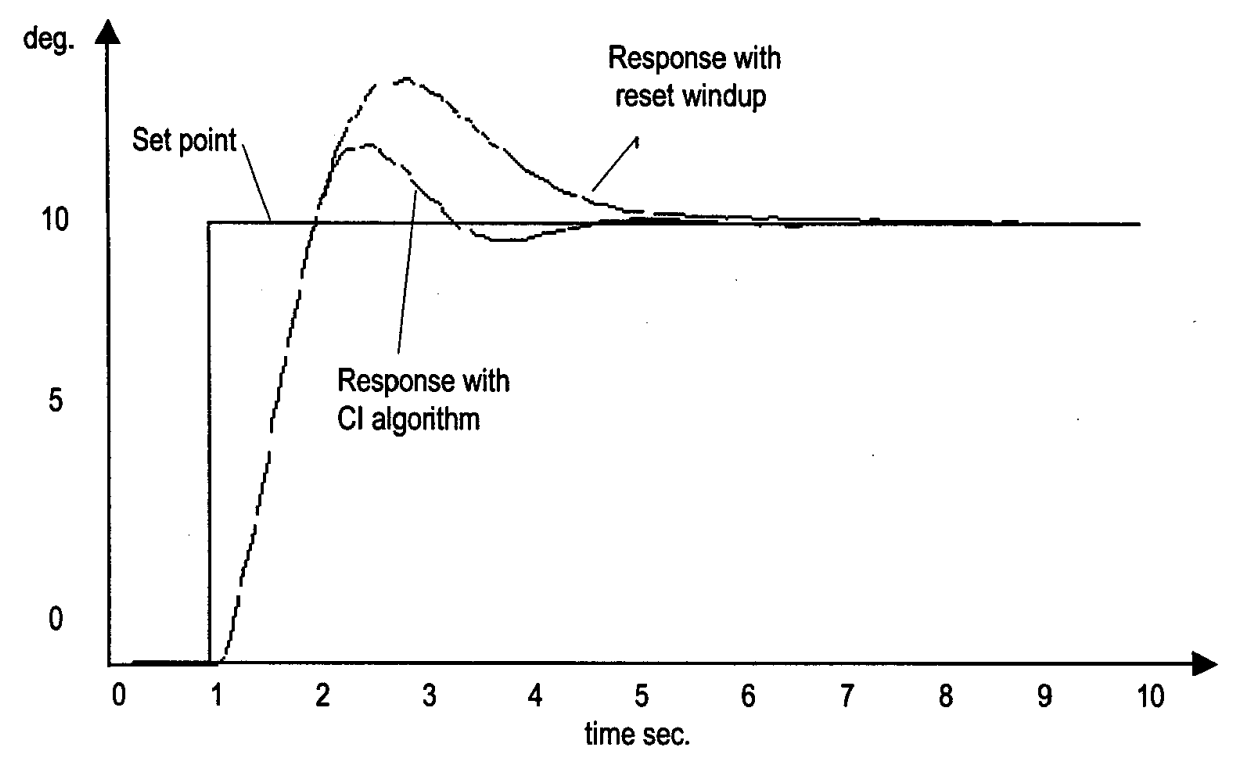

Fig.6. The response to a $10 \mathrm{deg}$. command input with $\mathrm{CI}$ and without CI.

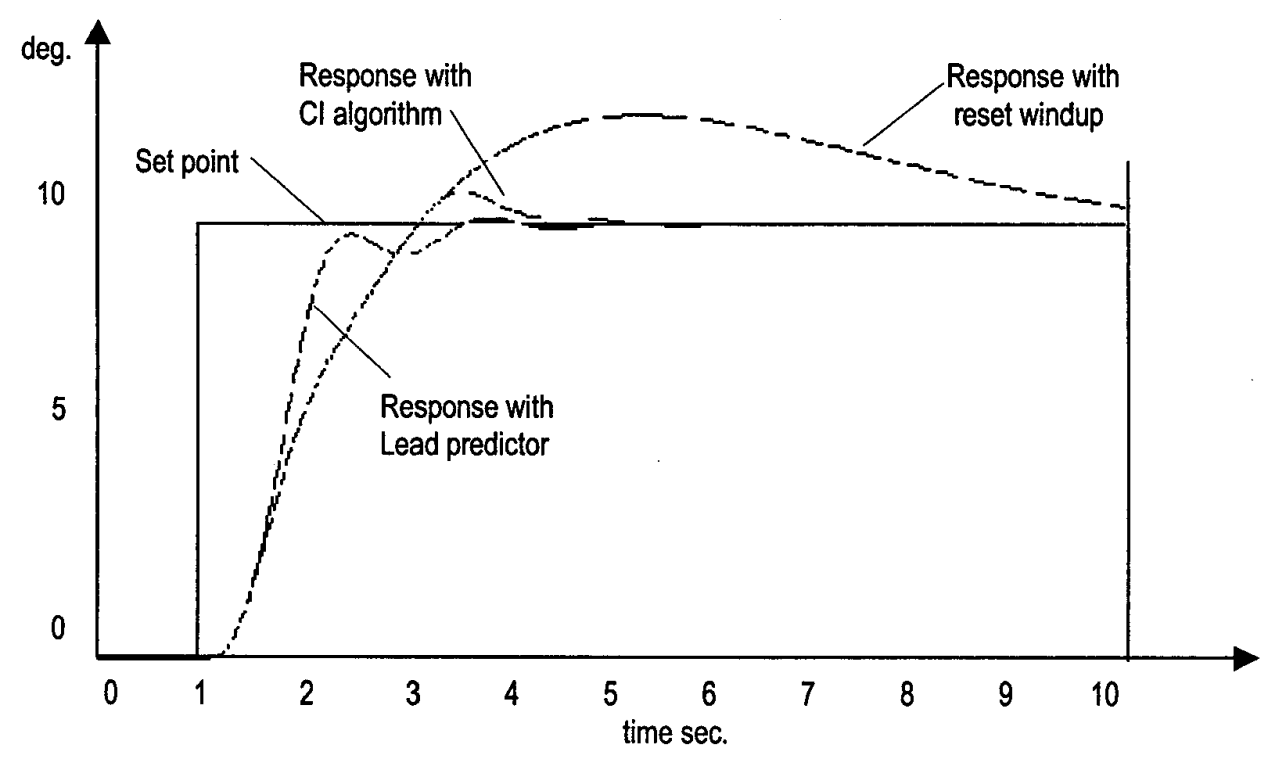

Fig. 7. The response to a $10 \mathrm{deg}$. command input with $\mathrm{CI}$, without $\mathrm{CI}$ and with a lead predictor compensator 